\title{
Miscel·lània
}

\author{
MARINA GARCÍA-GRANERO 1
}

\section{Injusticias de género en tiempos de neoliberalismo. El planteamiento de Nancy Fraser}

\section{Gender Injustices in Neoliberal Times. On Nancy Fraser's Proposal}

\begin{abstract}
RESUM
El artículo se propone elaborar una crítica al neoliberalismo desde una perspectiva de género, empleando la teoría de la justicia de Fraser como marco analítico. Tras recoger la narrativa histórica que Fraser elabora en torno al feminismo de la segunda ola y sus relaciones con el capitalismo, se realiza un análisis pormenorizado de las características del modelo neoliberal según los criterios de redistribución, reconocimiento y representación de la teoría de la justicia de Fraser. Finalmente, se analizan las críticas que diversas teóricas, entre las que destaca Fraser, han realizado a un supuesto feminismo neoliberal, el cual deja intactas las estructuras de dominación económicas y promueve únicamente el empoderamiento de una minoría privilegiada de mujeres.
\end{abstract}

Palabras clave: Nancy Fraser, justicia, género, neoliberalismo, redistribución, capitalismo, feminismo.

\begin{abstract}
This article aims to elaborate a critique of neoliberalism from a gender perspective, using Nancy Fraser's theory of justice as an analytical framework. I first present Fraser's historical narrative of second-wave feminism and its relations with capitalism. Then I develop a detailed assessment of the current neoliberal model, according to the criteria of redistribution, recognition and representation from Fraser's theory of justice. Lastly, I examine the critiques that some feminist authors, Fraser among them, have leveled against a so-called neoliberal feminism, which safeguards the economic structures of domination and only promotes the empowerment of a privileged minority of women.
\end{abstract}

Keywords: Nancy Fraser, justice, gender, neoliberalism, redistribution, capitalism, feminism.

\section{SUMARI}

- Introducción. 1.- La astucia de la historia: del Estado de Bienestar a la crisis neoliberal. 2.- Neoliberalismo e injusticias de género. 3.- ¿Un feminismo neoliberal? - Conclusiones. Referencias bibliográficas.

1 Departamento de Filosofía, Universitat de València. Marina.Garcia-Granero@uv.es. Este estudio se ha podido realizar gracias a una ayuda a contratos FPU del Ministerio de Ciencia, Innovación y Universidades (Referencia: FPU15/04085). 


\section{Introducción}

El neoliberalismo es el sistema que, tras destruir las fronteras que el liberalismo había establecido, somete y evalúa todos los ámbitos de la sociedad según los imperativos del mercado. Se alteran las fronteras entre la producción y la reproducción, entre el mercado, el Estado y la sociedad civil, y entre lo nacional y lo global. Contrariamente al liberalismo, el neoliberalismo no crea un espacio aparte para los imperativos del mercado, separados de la relativa integridad del resto de esferas, sino que los nuevos modos de subordinación del mercado fagocitan hasta el último aspecto del Estado y del mundo de la vida. El neoliberalismo hace suyo el proyecto ideológico de traducción de todas las esperanzas emancipatorias en términos amistosos con el capitalismo, y, como veremos en este artículo, el feminismo no ha sido una excepción. La igualdad pasa a ser entendida de acuerdo con una visión idealizada del intercambio mercantil, en la que agentes independientes intercambian libremente mercancías equivalentes ${ }^{2}$.

Un feminismo que se pretenda realmente emancipador no puede pasar por alto la letal combinación de austeridad, libre comercio, deuda predatoria y trabajo precario y mal pagado, que resulta característica del actual capitalismo neoliberal, y que repercute especialmente en la calidad de vida de las mujeres. Por ello, en el presente artículo, recogeremos la crítica de la filósofa estadounidense Nancy Fraser al neoliberalismo, empleando como piedra de toque su teoría de la justicia. Fraser es una de las teóricas contemporáneas que más ha trabajado la situación del feminismo en la era "postsocialista» (Fraser, 1995) y que más ha incidido en la importancia de la tematización de la economía política por parte de la teoría feminista.

Comenzaremos examinando la narrativa histórica que Fraser elabora en torno al feminismo de la segunda ola y sus relaciones con el capitalismo. A continuación, realizamos un análisis pormenorizado de las características del actual modelo de gobierno neoliberal según los criterios de redistribución, reconocimiento y representación de la teoría de la justicia de Fraser. Finalmente, disputaremos las premisas de un presunto «feminismo neoliberal» y recogeremos las críticas que diversas teóricas, entre las que se encuentra Fraser, han dirigido a esta cooptación del feminismo por parte del neoliberalismo.

\section{La astucia de la historia: del Estado del bienestar a la crisis neoliberal}

Con la ventaja que proporciona la retrospectiva, Fraser analiza la evolución del feminismo de la segunda ola partiendo de la década de los setenta del siglo xx hasta el presente, y periodizándolo como «un drama en tres actos» (Fraser, 2015a: 17), estando cada uno de ellos relacionado con un momento de la historia del capitalismo. Esta periodización fue publicada en un primer momento en un artículo en la New Left Review (Fraser, 2009) y fue retomada con más detalle posteriormente en su último 
libro, Fortunas del feminismo (Fraser, 2015a). Al contextualizar los cambios en las energías feministas, Fraser pretende elucidar cómo podríamos revitalizar la teoría y la práctica de la igualdad entre los sexos en las condiciones socioeconómicas actuales.

El primer acto es el del «feminismo insurgente», el de los comienzos del feminismo de la segunda ola en la época de posguerra. Según Fraser, este es el feminismo que realizó la crítica radical de la socialdemocracia. El contexto o paradigma económico era el capitalismo gestionado por los Estados del Bienestar, que empleaban herramientas keynesianas para suavizar los ciclos de auge y caída endémicos del capitalismo, entre ellas la inversión en infraestructuras, la tributación redistributiva, las prestaciones sociales, la reglamentación empresarial, la nacionalización de los sectores industriales clave y la desmercantilización de los bienes públicos. Estas herramientas de gestión económica garantizaban el pleno empleo de los varones, al mismo tiempo que los movimientos sindicales, satisfechos con el sistema de redistribución, institucionalizaban la solidaridad nacional entre las clases (Fraser, 2015a: 19).

Según Fraser, el movimiento por la liberación de las mujeres empezó como cuestionamiento de este acuerdo entre clases, que descansaba en una serie de exclusiones tanto de género como etnorraciales, sin olvidar la explotación neocolonial externa. Dicho feminismo insurgente, surgido del fermento que rodeaba a la Nueva Izquierda ${ }^{3}$, cuestionó las exclusiones de la socialdemocracia y desveló el androcentrismo subyacente a los modos en que se habían articulado los Estados de Bienestar. Además, exigió que las luchas por la distribución socioeconómica integraran la igualdad entre los sexos, y su variante más radical se centró en politizar lo personal para incluir el trabajo doméstico, la sexualidad y la reproducción, y así poder transformar la sociedad desde la raíz.

El segundo acto es el del «feminismo domesticado», el feminismo que en la década de los 80 realiza el tránsito del paradigma de la redistribución al paradigma del reconocimiento en la era de la identidad. Una década de dominio conservador en buena parte de Europa Occidental y Norteamérica, culminada por la caída del comunismo, insufló nueva vida a las ideologías del libre mercado. En este contexto se discutió la legitimidad del uso del poder público para controlar las fuerzas del mercado. Fraser emplea la expresión «la astucia de la historia» para designar la coincidencia de las luchas por el reconocimiento, que pusieron el acento en la política cultural de la diferencia, con el auge del neoliberalismo, que acrecentó las desigualdades sociales en materia económica (Fraser, 2015a: 245). Si la anterior generación había intentado rehacer la economía política, ésta se centraba en la cultura, en los cuidados, la violencia sexual y las disparidades entre sexos en la representación política. Sin duda, Fraser reconoce la importancia de estas luchas,

3 Nueva Izquierda o New Left fue un movimiento estadounidense de la década de los sesenta. Herbert Marcuse es considerado el padre ideológico del movimiento. Se denomina Nueva Izquierda en oposición a la "vieja izquierda» marxista centrada en cuestiones de clase y de trabajo, y nace como respuesta y oposición al autoritarismo soviético. En su lugar, la Nueva Izquierda se centró especialmente en la contracultura de los derechos civiles, homosexuales, la crítica de los roles de género y en problemas bioéticos como el aborto. Abordó una perspectiva internacional, que resonó en países como Japón, Brasil y Australia y dejó una huella que hasta el día de hoy se siente en varios sectores de la sociedad norteamericana y en personalidades como Noam Chomsky (Gosse, 2005). 
pero su crítica está dirigida a la resignación frente a la economía capitalista. A juicio de Fraser, aunque la primera intención de estos feminismos culturales fuese ampliar el abanico de sus luchas, acabó teniendo lugar una desatención de la economía política, en el preciso momento en que el avance neoliberal requería redoblar la atención sobre la crítica de la economía política.

Fraser denomina «feminismo domesticado» a aquel que, en consonancia con el espíritu postsocialista de la época, redefinió la justicia de género únicamente como proyecto dirigido a reconocer la diferencia (Fraser, 2015a: 21). El feminismo domesticado no profundizó en el imaginario socialista atendiendo al reconocimiento, sino que, cayendo presa del espíritu del tiempo, lo desplazó. En lugar de abarcar tanto la redistribución como el reconocimiento, intercambiaron un paradigma incompleto por otro: un economicismo truncado por un énfasis desproporcionado en la cultura (Fraser, 2015a: 254). Tras la fragmentación de la segunda ola, se produjo la incorporación selectiva y parcial de algunas de sus líneas, al mismo tiempo que los intereses culturales ganaban importancia sobre los económicos, lo cual coincidió con los intereses de la hegemonía neoliberal.

En el tercer acto, que se corresponde con la época actual, se estaría produciendo una revitalización del feminismo socialista, preparado para denunciar las injusticias derivadas de la hegemonía neoliberal. Defiende Fraser que se debería aprovechar el momento de crisis del capitalismo financiero para reactivar la promesa emancipadora del feminismo, cuya reorientación podría marcar el comienzo de una transición hacia una nueva forma de organización social (Fraser, 2015a: 244). Por ello, lo denomina el «feminismo resurgente»: este sería un feminismo que recuperaría la radicalidad inherente a toda lucha por la igualdad y que aprovechase la actual crisis de legitimación capitalista en la era neoliberal para abogar por un cambio sistémico de la sociedad en su conjunto (Fraser, 2015b).

Conviene apuntar que este análisis ha recibido críticas, entre las que destaca las de Nanette Funk (2013). Según Fraser, esta reconstrucción es únicamente una «mirada amplia» sobre «los amplios contornos y el significado general del feminismo de la segunda ola» (Fraser, 2009: 97). Ahora bien, la crítica de Funk radica en que Fraser extrapola erróneamente una posición minoritaria del feminismo como corriente principal, pues este relato del feminismo de segunda ola "como fundamentalmente anticapitalista es inexacto, y confunde una minoría del movimiento (...) con el movimiento en general» (Funk, 2013: 179). De entre las puntualizaciones de Funk, me parece pertinente señalar con ella que en Norteamérica:

La forma dominante del feminismo de la segunda ola a mediados de siglo era el feminismo liberal que criticaba el Estado, pero se centraba en cambiar las políticas y las leyes, modificando, pero no criticando fundamentalmente, el capitalismo. El derecho de las mujeres al aborto y las leyes anti-discriminatorias fueron cuestiones importantes del feminismo de segunda ola, pero eran criticismos al Estado y a sus leyes, y estos cambios no eran generalmente considerados incompatibles con un capitalismo robusto (Funk, 2013: 181).

El feminismo liberal siempre ha colocado el énfasis en el derecho de las mujeres a participar en la esfera pública en términos igualitarios con los hombres, y estos 
parámetros de igualdad serían, según esta corriente, alcanzables dentro del capitalismo. Podemos conceder que, efectivamente, Fraser proyecta una idea exagerada de la magnitud del feminismo radical o socialista, representándolo como mayoritario, cuando en realidad, el feminismo liberal siempre ha sido hegemónico en Norteamérica. Con todo, el sentido de la narrativa histórica de Fraser permanece el mismo, y es que sea cual fuere el peso del feminismo socialista o «insurgente» del que ella nos habla, éste menguó progresivamente en los años 80 y 90, en el mismo momento en que Occidente transitaba a la era post-socialista y neoliberal. El objetivo de esta cartografía del imaginario feminista es ayudarnos a determinar qué debemos descartar y qué preservar para las luchas futuras, y entre aquello que debemos recuperar se encuentra la crítica explícita de la economía política y el discernimiento de las pretensiones de validez de los distintos modelos económicos. No hemos de olvidar que las estructuras de dominación económicas contribuyen a la dominación de género.

Ahora que el sistema financiero se tambalea y las promesas de los modos de gobierno neoliberales se están demostrando falsas, el contexto de crisis actual ofrece un escenario óptimo para un feminismo resurgente, un feminismo que, a mi juicio, se percate de que la igualdad de oportunidades que promete el feminismo liberal no es suficiente ${ }^{4}$, y que hace falta recuperar la ambición transformadora de la sociedad, para asegurar unas mejores condiciones de vida para todas las mujeres, y el deseo de transición hacia un mundo mejor y más justo.

A continuación, realizaremos una evaluación del neoliberalismo según la teoría de la justicia de Fraser. Entendiendo que la igualdad sólo puede ser entendida en un sentido multidimensional, el feminismo ha de emplear un marco conceptual que tematice todas las esferas y ámbitos de la sociedad, y es por ello que Fraser defiende una teoría tripartita de la justicia que atiende al mismo tiempo la redistribución, el reconocimiento y la representación. En la siguiente sección, me propongo mostrar que el neoliberalismo es un modelo económico que no satisface ninguno de los criterios de justicia que marca Fraser, sino que propicia la falta de distribución, el reconocimiento fallido y la ausencia de representación.

\section{Neoliberalismo e injusticias de género}

En un primer momento, Fraser elaboró una teoría delajusticia en dos dimensiones, con el fin de superar la dicotomía entre el paradigma de la redistribución, asociado a la justicia socioeconómica, y el paradigma del reconocimiento, que se centra en la justicia cultural y las demandas de reconocimiento de las grupos oprimidos y excluidos (Posada Kubissa, 2015). Según Fraser, el feminismo cultural convirtió «el lenguaje y la subjetividad en los focos preferidos de la crítica feminista» (Fraser, 2015a: 27), y en realidad, si queremos alcanzar una verdadera transformación no debemos dejar inalteradas las estructuras materiales de la sociedad. El giro hacia el reconocimiento debería complementarse con la política de la redistribución del

4 Como muy agudamente expresó Bonnie Kreps en 1968, no creemos que la opresión de las mujeres se termine dándoles un trozo más grande del pastel, porque el pastel en sí mismo está podrido. 
feminismo socialista. Ahora bien, esta teoría devino tridimensional a partir del 2005, cuando Fraser decide abordar la cuestión de la representación en un mundo globalizado (Fraser, 2005).

Esta teoría tiene como objetivo servir como teorización crítica capaz de clarificar desde una perspectiva sistémica las virtualidades y las carencias de los distintos modelos de organización social. Para Fraser, el feminismo es un movimiento social en la más estricta tradición democrática, una «contraesfera pública» empeñada en abrir un nuevo espacio discursivo y colectivo en el que las mujeres tomen la palabra para expresar sus propias necesidades (Guerra, 2011: 318). De este modo, y frente a la desafección política propia del neoliberalismo, Fraser aboga fervientemente por la recuperación de la vocación política, la intensificación de los debates y la preocupación por la justicia social.

El horizonte de igualdad que ha de ser conquistado será la paridad participativa: que los distintos grupos puedan interactuar como iguales en la vida social. Por ello, tanto las medidas de política de clase (redistribución) como las medidas de política de identidad/estatus (reconocimiento) y política representativa (representación) han de estar orientadas a garantizar la paridad de participación en la interacción social. Se trata de desmantelar los obstáculos institucionales que impiden a algunas personas participar a la par que otros como interlocutores plenos (Fraser, 2015a: 225).

La igualdad puede verse amenazada en un sentido multidimensional, esto es, una persona puede sufrir opresión por parte de las tres esferas: la economía, la cultura o la política. Pero lo que Fraser nos quiere mostrar es que no podemos tratar de erradicar una injusticia sin elucidar el ámbito en que nace y se perpetúa. Hemos de ser capaces de distinguir si se trata específicamente de una injusticia por mala distribución, reconocimiento fallido, o ausencia de representación. A continuación, analizaremos si el neoliberalismo cumple o no los criterios de justicia de la teoría de la justicia de Fraser.

\subsection{Falta de redistribución}

La primera variable de la teoría de la justicia de Fraser es la redistribución, que se corresponde a la dimensión económica. El concepto clave es el de «clase social». Las clases son un modo de diferenciación social arraigado en la estructura políticoeconómica de la sociedad y representan un obstáculo para la paridad participativa, en la medida en que las desigualdades económicas impiden que las personas desprovistas de recursos interactúen en términos de igualdad con aquellas que los acumulan.

Las injusticias en economía política tienen como consecuencia la creación de clases trabajadoras explotadas, entre las que destacan las mujeres en la medida en que sufren tanto la precariedad en el trabajo remunerado como la extenuación en el trabajo doméstico no remunerado (Vélez Bautista, 2009). Estas injusticias se corrigen mediante políticas redistributivas que conlleven reformas estructurales profundas, similares a las antaño realizadas por el capitalismo gestionado por el Estado del Bienestar, que, con el objetivo de promover la igualdad y la solidaridad 
entre las clases, dirigieron la economía política en los tiempos de posguerra para asegurar una distribución equitativa de bienes, especialmente la renta y los puestos de trabajo. Y para atender a la extenuación a causa del trabajo doméstico, también sería necesaria la redistribución de este tipo de trabajo y del tiempo libre por parte de todos los miembros de la familia.

No obstante, desde los años ochenta, la hegemonía neoliberal construye un discurso que intersecta las políticas familiares y del bienestar con la oposición a los impuestos, basándose en asunciones erróneas y en prejuicios sobre las necesidades y los derechos de las personas (Fraser, 1989a). Fraser fue una de las primeras teóricas en destacar que los ataques contra el Estado de Bienestar serían, principalmente y, antes que nada, ataques contra las mujeres (Fraser, 1989b: 144), ya que los recortes en la asistencia pública en nombre de la austeridad perpetúan el orden de género en nuestras sociedades.

Bajo los mandatos de Ronald Reagan (1981-1989) y George H. W. Bush (1989-1993) seinauguró un imaginario político quela filósofa denomina el «salario antisocial» (the antisocial wage) (Fraser, 1993: 10). Tanto Reagan como Bush criticaron los impuestos y el gasto gubernamental, exaltando, en su lugar, una visión reduccionista del nivel de vida como dependiente únicamente de los ingresos personales o familiares. Esta visión omitía todos aquellos bienes públicos y servicios que anteriormente convergían en la idea de "prestaciones» (the social wage) y otros elementos claves que repercuten en la calidad de vida de las personas, como la educación, la sanidad, la seguridad de las ciudades, la sostenibilidad medioambiental y la cultura pública. La privatización de servicios de primera necesidad, como el agua y la electricidad, y el deterioro o privatización de los servicios públicos, va en contra de los intereses de las familias con recursos limitados, y especialmente de las mujeres, dado el contexto de feminización de la pobreza.

Nos enfrentamos a una ideología de la privatización que en apariencia es neutra con respecto al género. Dicha ideología divide la población en dos clases: por un lado, aquellos que son responsables, proactivos y capaces de gestionar su vida por sí solos, y por otro, aquellos que son pasivos o incompetentes, y que necesitan tutelaje público. Mientras que los miembros del primer grupo invierten sus ahorros y gestionan autónomamente sus planes de pensiones, los segundos reniegan de su responsabilidad, dependen del sistema de pensiones público y ceden el control de sus vidas al gobierno.

Esta demonización de la dependencia respecto al Estado social constituyó un ataque a las labores del cuidado y la provisión pública, y supuso al mismo tiempo una revalorización el trabajo remunerado y la mercantilización. A la larga, se criticó el derecho (entitlement) a subsidios para familias de bajos ingresos. Visto desde una perspectiva material, la situación económica de mujeres y niños/as en situación de pobreza empeoró, destruyéndose, además, los mecanismos que posibilitaban que una mujer abandonase a un marido violento o un empleo explotador. Desde la perspectiva simbólica, el ataque a los subsidios familiares lanzó el mensaje de que las beneficiarias de dichos programas eran «gorronas» (scroungers) que recibían dinero a cambio de nada, ya que, en una sociedad mercantilizada, las labores del 
cuidado y de crianza no gozan de reconocimiento social (Fraser y Bedford, 2008: 227). La dependencia legítima es la privada, la de una mujer respecto a su marido sustentador, mientras que la dependencia del tesoro público es ilegítima (Fraser y Gordon, 1994). Persuadidos por el discurso de Reagan y Bush, gran parte de la ciudadanía comenzó a renunciar a las prestaciones, para no ser considerados ciudadanos/as de segunda categoría.

La nueva ideología promueve una concepción mercantilizada de la ciudadanía. La única actividad con reconocimiento social es el trabajo remunerado, sin importar lo mal pagado o humillante que sea dicho empleo. Sólo éste confiere la independencia y el estatuto de una ciudadanía completa. De este modo, se obliga a las mujeres a incorporarse al trabajo remunerado, al mismo tiempo que disminuye la protección social y se mantienen inalteradas las normas de la reproducción social $^{5}$ (Fraser, 2015a: 14).

El neoliberalismo es un modelo económico que perpetúa las desigualdades de clase. No sólo acrecienta las disparidades entre las personas ricas y aquellas que no tienen recursos, sino que favorece la creación de clases trabajadoras explotadas, entre las que destacan las mujeres. Las mujeres han de soportar un trabajo fuera del ámbito doméstico, precario y mal pagado, al mismo tiempo que su carga de trabajo no-remunerado se ve acrecentada, tras la desaparición de los servicios públicos encargados de favorecer los cuidados, tanto de infantes como de personas dependientes.

Y es que las mujeres no acceden al mercado con los mismos recursos y la misma movilidad que los varones, y por ello, no pueden competir en igualdad de condiciones en el ámbito público. Su acceso se ve condicionado por lo que la economista feminista Ingrid Palmer denomina «el impuesto reproductivo» ${ }^{6}$ que se realiza en el ámbito doméstico, y que es creado a medida que el Estado se desentiende de los costes de reproducción y manutención de la vida (Palmer, 1995). De hecho, los informes internacionales en temas de igualdad de las principales agencias, como la ONU o el Banco Mundial, no reflexionan sobre el gran volumen de trabajo no pagado que realizan las mujeres en el mundo, en sus roles de cuidadoras, madres, esposas e hijas. Se promueve así «un discurso igualitarista sin una verdadera transformación de las jerarquías patriarcales; manteniendo con ello la desigualdad» (Reverter-Bañón, 2017: 312).

5 Mediante el término «reproducción social», Fraser designa la creación y el mantenimiento de los vínculos sociales, por ejemplo, los vínculos entre las generaciones -el nacimiento y la crianza de los / as niños / as y el cuidado de las personas mayores-, los vínculos familiares y los vínculos de amistad, los vínculos vecinales y comunitarios, etc. La carga de responsabilidad de la reproducción social ha caído mayoritariamente en las mujeres. Si bien la reproducción social es absolutamente esencial para el mantenimiento y el progreso de las sociedades, ha sido menospreciada e infravalorada, en parte por su condición de «trabajo no remunerado» y su adscripción al ámbito privado y doméstico (Leonard y Fraser, 2016).

6 Según Palmer, el mantenimiento y cuidado de las familias puede considerarse como un impuesto cuyo pago se exige a las mujeres antes de que puedan iniciar cualquier otra actividad generadora de rentas (Palmer, 1995). 


\subsection{Reconocimiento fallido}

La segunda variable de la teoría de la justicia de Fraser es la del reconocimiento, que se corresponde con la dimensión cultural. Este eje tiene como objetivo tematizar y denunciar las jerarquías de valor cultural institucionalizadas, que niegan a las personas la posición necesaria para interactuar en términos de igualdad.

Si en el ámbito de la redistribución, la variable analítica clave era la de la clase, en este caso Fraser recupera de Max Weber la noción de estatus para designar las diferencias de honor, prestigio y respeto social que se derivan de los patrones institucionalizados de valor cultural (Fraser, 2006: 21-26). De este modo, se plantea el problema del reconocimiento como una cuestión de justicia, evitando la psicologización y la aceptación acrítica de las diferencias. Fraser no defiende una política del reconocimiento basada en la identidad, sino en la marca jerárquica, el estatus, que se coloque sobre dicha identidad. Se tematizan las consecuencias que conlleva la pertenencia a un colectivo (mujeres, personas negras, migrantes, clase obrera, etc.). Rechaza pensar el paradigma del reconocimiento como mera «realización personal» (Fraser, 2006: 35) y su enfoque pragmatista imposibilita además la proposición de que todo el mundo necesita siempre que se reconozcan sus caracteres distintivos, incluso cuando esta distinción no tenga repercusiones sociales en materia de reconocimiento. La ausencia de reconocimiento se aprecia en la desvalorización de algunos actores sociales como inferiores, excluidos, diferentes o sencillamente invisibles (Fraser, 2006: 36).

Si hablamos del paradigma del reconocimiento en el caso del género, el modo de corrección del estatus consiste en la revalorización cultural de todo aquello que al haber sido conceptualizado como femenino goza de menor estatus que la contrapartida otorgada a los varones. Por ejemplo, las tareas de los cuidados frente al trabajo remunerado: las feministas y sus aliados deberían defender una forma de vida que reste importancia al trabajo remunerado y dé valor a actividades no mercantilizadas, como los cuidados, de manera que dichas actividades pasasen a ser componentes valorados de una buena vida para todos (Fraser, 2015a: 261).

La combinación de los paradigmas de la redistribución y el reconocimiento tiene como objetivo permitir un análisis que atienda tanto a la desigualdad económica como a la diferenciación por razón de estatus, sin desatender ninguna de las dos variables, pues en numerosos casos son interdependientes. El caso de la injusticia de género ejemplifica cómo ambos órdenes de subordinación se interrelacionan, ya que «sólo un enfoque que repare la devaluación de lo «femenino» precisamente dentro de la economía y en otros ámbitos, puede llevar a una redistribución seria y a un auténtico reconocimiento» (Fraser, 2006: 66). Los miembros de una clase pueden sufrir discriminaciones culturales, pero estas injusticias no se producen autónomamente de la economía política, sino que se derivan de ella (Fraser, 1997: 27-28). Pensemos, por ejemplo, en cómo los oficios y profesiones típicamente ejercidos por mujeres $3 / 4$ por ejemplo, los servicios de limpieza (camareras de hotel), el cuidado de niños/as y personas dependientes (puericultura, enfermería), etc., $3 / 4$ gozan tanto de menor estatus como de menor remuneración económica que las 
profesiones típicamente ejercidas por hombres, o también en cómo una persona negra o gitana puede sufrir discriminación racial en su búsqueda de empleo y en la posterior remuneración que reciba por él. Por ello, se ha de luchar por desmantelar las jerarquías de estatus tradicionales que impiden la plena participación de mujeres y otros colectivos desprestigiados en la vida social.

El género es, pues, una forma híbrida de diferenciación que depende tanto de la estructura económica como del orden simbólico y cultural. Hay desigualdades de género en materia económica, por ejemplo, la separación de la esfera pública productiva y la esfera privada reproductiva con su consecuente división del trabajo, la brecha salarial, la explotación laboral, la doble jornada, etc., y también en materia cultural, como el androcentrismo, la violencia de género, el lenguaje sexista, los roles y estereotipos de género, la devaluación de la feminidad y la maternidad. No obstante, para no caer ni en el economicismo truncado ni en el culturalismo truncado, es necesario analizar las desigualdades mediante el dualismo perspectivista.

La ideología neoliberal reduce la igualdad a una visión idealizada del intercambio mercantil, en el que agentes económicos independientes intercambian libremente mercancías equivalentes. Promete mayores ingresos a quien más trabaje, ingresos que en teoría irían acompañados de mayor dignidad e independencia respecto a la autoridad tradicional. Esta visión se mantiene ciega ante la coerción y la desigualdad que realmente vertebran los intercambios en el mundo real. Se celebra la elección individual y el logro meritocrático, al tiempo que se ocultan los mecanismos estructurales e ideológicos que realmente condicionan las elecciones de las personas según su sexo (De Miguel, 2015: 9). El marco axiológico neoliberal vende un discurso según el cual la promoción personal siempre dependerá de los méritos que cada persona recolecte, enmascarando tanto las diferencias en los puntos de partida como las dinámicas de opresión y discriminación que impiden el correcto reconocimiento de las personas (Fraser, 2015a: 226). Por ello, deja intactas las jerarquías de valor cultural institucionalizadas (androcentrismo, heterosexualidad normativa, supremacía blanca, etc.) que niegan a muchas personas la posición necesaria para interactuar en términos de igualdad.

Se trata pues de distinguir dos formas de entender la igualdad, una idea de igualdad neoliberal y mercantilizada, o una idea de igualdad propia de una democracia radical, que busca la paridad participativa de todas las mujeres. El marco neoliberal es perverso en la medida en que niega todas las desigualdades estructurales del orden del estatus, de modo que estas estructuras quedan intactas. Concluimos, por tanto, que el neoliberalismo no soluciona, sino que perpetúa, la falta de reconocimiento.

\subsection{Ausencia de representación y des-enmarque}

A partir de 2005, Fraser incorpora una tercera dimensión a su teoría de la justicia. La representación es la más intrínsecamente política, en la medida en que hace referencia a la constitución tanto de los Estados como del derecho internacional, a las normas y procesos de tomas de decisiones por las que ambos se estructuran. Fraser observa que no sólo está en disputa la cuestión de cuáles son los asuntos 
de justicia, es decir, qué derechos posee la ciudadanía, sino también quién cuenta cómo ciudadanía y cuál es la comunidad pertinente: «no sólo está en disputa el fondo de la justicia sino también el marco» (Fraser, 2015a: 31). La representación es una cuestión de pertenencia social. Está en juego la inclusión o la exclusión de aquellos que podrían verse llevados a presentar reivindicaciones de justicia, quién está incluido o excluido del círculo de las personas con derecho a una distribución justa y al reconocimiento recíproco.

La falta de representación se da cuando los procedimientos de toma de decisión niegan, tanto a personas como colectivos, la posibilidad de participar a la par que otras en interacción social y en las reclamaciones de justicia. Como ejemplo, destaca el caso de los migrantes indocumentados que, al verse privados de la posibilidad de presentar reivindicaciones de justicia, se convierten en no-persona. Similar a lo que Hannah Arendt denominó «el derecho a tener derechos», la carencia de marco conlleva una muerte política.

La cuestión del marco es pertinente durante el análisis de los procedimientos y tomas de decisión realizadas que surgen dentro de una comunidad política, para evitar que decisiones sesgadas neutralicen las disparidades de voz de la ciudadanía y las necesidades de grupos desatendidos (Blanco Brotons, 2017). En este sentido, el establecimiento de cuotas de mujeres en las listas electorales es una respuesta a este tipo de falta de representación. Pero el objetivo principal de Fraser a la hora de plantear la cuestión de la representación es aclarar los enfrentamientos causados por la globalización y denunciar los «marcos injustos». La globalización en su vertiente neoliberal ha tenido como consecuencia que el escenario donde se desarrollan las luchas por la distribución y el reconocimiento haya cambiado: «Dada la creciente relevancia de los procesos transnacionales y subnacionales, el Estado soberano westfaliano ya no sirve como la única unidad o ámbito de justicia» (Fraser, 2006: 84).

Los procesos que condicionan la calidad de vida de la ciudadanía de un Estado concreto sobrepasan con frecuencia las fronteras territoriales, especialmente en el contexto de la globalización neoliberal. Si bien las políticas internas de Estados Unidos, en tanto que potencia mundial, tendrán repercusiones inestimables en el resto de países del mundo, el resto de la ciudadanía global carece de marco de acción e interacción. Las empresas multinacionales, los especuladores en divisas y los grandes inversores gozan de un poder cada vez mayor en la economía financiarizada, al mismo tiempo que se les exime del control democrático. Por ello, un marco westfaliano que atienda únicamente a las políticas internas protegerá a los Estados depredadores y a los poderes privados más poderosos, y mantendrá incuestionadas e inalteradas las estructuras de gobernanza de la economía mundial y sus condiciones de interacción explotadoras (Fraser, 2015a: 231).

La lucha por la representación es la lucha contra el sometimiento político, o más concretamente, en el contexto neoliberal, la lucha contra el sometimiento de las personas y de los gobiernos al poder de los mercados. Una política de representación adecuada no sólo debe asegurar la participación ordinaria (nacional), sino también establecer un marco poswestfaliano que cuestione y revise la división del poder y la autoridad entre los diferentes agentes estatales y también los económicos. Esta política 
de representación no está garantizada por el neoliberalismo. Perpetuar el marco westfaliano en el contexto neoliberal y de economía globalizada es, en realidad, un poderoso instrumento de injusticia, ya que inmuniza las superpotencias transnacionales.

Como vemos, la teoría tripartita de Fraser busca un enfoque holístico, concreto e integrado al mismo tiempo, que pueda reparar las injusticias de todas las esferas. Se buscaría siempre que fuese posible la reparación transversal (Fraser, 2006: 80), pero sin perder de vista el ámbito específico en el que nace y se propaga cada mecanismo de subordinación.

Hemos visto que el neoliberalismo no satisface ninguno de los criterios de justicia de la teoría de Nancy Fraser. Los modos de gobierno neoliberales fomentan la ausencia de redistribución, el reconocimiento fallido y la privación de representación. Ante este diagnóstico, nos preguntamos: ¿cómo ha de trabajar el feminismo del presente y del futuro de cara a cambiar estas injusticias? Sin duda, un feminismo excesivamente dependiente de los fundamentos analíticos y conceptuales del marxismo deja sin respuesta aspectos simbólicos y culturales del androcentrismo y de la heterosexualidad normativa, que son esenciales para la derogación del sistema patriarcal. Pero cuando los estudios de género obvian en sus análisis las estructuras económicas, pueden acabar sirviendo a los intereses del neoliberalismo. Por ello, Fraser recupera la expresión de Eisenstein (2005) y habla del «peligroso vínculo» entre feminismo y neoliberalismo, que analizaremos en la última sección del artículo.

\section{3. ¿Un feminismo neoliberal? ${ }^{7}$}

De acuerdo con la retrospectiva analizada en la primera sección de este artículo, Fraser augura la revitalización de un feminismo en el que la emancipación vuelva a ser beligerante con las desigualdades económicas, y que vaya de la mano de la democracia participativa y la solidaridad social. Ahora bien, lejos de haberse realizado esta revitalización del feminismo socialista, los más recientes artículos de Fraser han tenido como objetivo diagnosticar el desarrollo de un peligroso vínculo entre feminismo, corporativismo y mercantilización.

Según Eisenstein (2005) y Fraser (2015a), el pensamiento feminista no ha sido meramente engañado por el neoliberalismo, sino que ha sido «seducido», creándose una aventura (liaison). Por su parte, Johnson destaca que no se trata únicamente de una aventura, sino que nos encontramos ante una nueva variante del feminismo, que contempla el neoliberalismo como complemento ideológico (Johnson, 2017), de modo que el propio feminismo acaba estando en venta.

Estas autoras critican el feminismo endorsado por figuras como Hillary Clinton o Sheryl Sanberg ${ }^{8}$, que animan a que las mujeres profesionales se atrevan a partici-

7 Desde mi punto de vista, un supuesto feminismo neoliberal no merece ser llamado feminismo, pero emplearé la expresión en la medida en que es significativa y nos sirve para designar la integración de premisas feministas dentro del neoliberalismo, que resulta en una idea de igualdad individualista y despolitizada.

8 Sheryl Sandberg es la actual directora ejecutiva de Facebook y autora del célebre libro Lean In: Women, Work and the Will to Lead (2013), y que ha sido traducido al castellano como Vayamos adelante: las mujeres, el trabajo y la voluntad de liderar. 
par y tomar la palabra en los consejos de dirección de las grandes empresas, como si el techo de cristal fuese el resultado de una falta de ambición por parte de las mujeres. Ahora bien, hemos de cuestionar qué mujeres son invitadas a participar, hasta qué punto las oportunidades que nos son dadas no dependen a su vez de las desigualdades sociales y de los distintos puntos de partida, y si acaso la meritocracia y la competitividad han sustituido a la justicia social (Littler, 2013).

El discurso de este «feminismo neoliberal» defiende una planificación cuidadosa de la carrera profesional y la maternidad para asegurar una mayor rentabilidad en el futuro (Rottenberg, 2013, 2017). Esta razón produce sujetos de los que se espera que se comporten de tal modo que maximicen su valor capital en el presente y mejoren su valor futuro a través de prácticas de emprendimiento, autoinversión, atracción de inversores, autosuficiencia y asunción de riesgos (Brown, 2015).

Se coloca el acento en la conciliación (balance) como ideal feminista, lo cual es paradójico si tenemos en cuenta la destrucción de las estructuras del Estado del Bienestar que facilitaban la conciliación. De modo que dicha conciliación es inalcanzable para quien no tiene los recursos económicos que la costeen (guardería, servicio doméstico, etc.). Además, no se presenta como alternativa un modelo de organización laboral y de colaboración familiar que posibilitase que no fuera necesario recurrir a la cadena de los cuidados. Parece que las mujeres, y sólo las mujeres, son responsables de confeccionar su propio equilibrio entre carrera profesional y familia9. Las profesionales suelen recurrir a migrantes pobres con contratos precarios que cuidan los hijos/as y limpian el hogar mientras ellas trabajan fuera de casa (Martí Gual y Poveda Rosa, 2009). Este sería un feminismo para el cual triunfar en el ámbito empresarial requiere necesariamente apoyarse en la explotación de otras personas. Cuando algunas mujeres son capaces de congelar sus óvulos, alquilar una gestante y contratar varias cuidadoras, ocurren nuevas formas de explotación de clase y de género. Se crea una «infraestructura invisible para todo el capital humano en desarrollo, maduro o agotado, niños, adultos, discapacitados y mayores» (Brown, 2015: 105). De este modo, el feminismo neoliberal contribuye a producir una pequeña clase de mujeres ambiciosas que invierten en sí mismas, gracias a otra gran clase de mujeres explotadas.

Esta visión no busca alcanzar la igualdad entre los sexos a través del movimiento colectivo en la política o la cultura, sino que se convierte en una cuestión de responsabilización, de desarrollo de capacidades, de promoción del acceso de las mujeres a todos los niveles de la jerarquía empresarial, y de fomento de las aspiraciones individuales y de las identidades emprendedoras. Sin duda, todos estos objetivos son valiosos -y esto es algo que Prügl (2014) insiste mucho en matizar-, pero el problema clave y la gran deficiencia de este giro neoliberal radica en la ausencia de cuestionamiento de las estructuras de poder, la erosión del ímpetu emancipatorio y la despolitización del feminismo. La cooptación del feminismo por parte de la retórica neoliberal conlleva un abandono de su naturaleza política (Medina-Vicent, 2018). El neoliberalismo promueve las respuestas individuales

9 Destaca la falta de interpelación a los hombres, incluso en temas tan clave como la conciliación. 
frente a las injusticias sociales, de modo que desaparece la conciencia colectiva de que la desigualdad de género es una opresión que atañe a todas las mujeres como clase sexual ${ }^{10}$. Las autoras críticas con esta visión animan a recuperar un feminismo que no sólo anime a triunfar en los comités directivos, sino que problematice la jerarquía del management y las flagrantes diferencias entre los puestos directivos bien pagados frente a la precarización de los contratos de la plantilla (alta rotación, salario mínimo, tiempo parcial, etc.).

Fraser acuñó la expresión «neoliberalismo progresista» para designar la alianza desarrollada en los Estados Unidos durante las tres últimas décadas entre las corrientes principales de los nuevos movimientos sociales -tales como el feminismo en su vertiente cultural, el antirracismo, el multiculturalismo y los movimientos $\mathrm{LGTB}^{11}$ - con los sectores de negocios de alta gama y las corporaciones de Wall Street, Silicon Valley y Hollywood. Esta alianza supuso la aceptación del marco cosmovisivo del capitalismo, que ganó carisma y poder de persuasión gracias a su defensa de ideales como la diversidad y el empoderamiento, creando así un espíritu que reconfigura los movimientos emancipadores en términos mercantilizados.

Ya hace muchos años, Fraser avisaba de que el movimiento para la liberación de las mujeres se había enredado con los esfuerzos neoliberales de políticos como Bill Clinton y las dinámicas del libre mercado (Fraser, 1993). El neoliberalismo progresista ha destruido la comprensión de la emancipación de las mujeres, antaño crítica con el capitalismo, abarcadora, antijerárquica, igualitaria y sensible a la clase social. Esto explicaría por qué conceptos feministas, como el de «empoderamiento», que anteriormente formaban parte de un proyecto colectivo de emancipación, se expresan cada vez más en términos individualistas y despolitizados.

El neoliberalismo coloniza hasta los umbrales más íntimos de la privacidad y la subjetividad, y por ello, es necesario recolocar los imperativos de la economía en el lugar en que pertenecen -el ámbito estrictamente económico-, y poner frenos a una ideología que fagocita todos los ámbitos vitales subsumiéndolos a los criterios de rentabilidad y competitividad. La tarea de la emancipación feminista no consiste en evaluar los modos de ser en términos de eficiencia o beneficio, sino en investigar las prácticas de todos los ámbitos -político, económico y social-, desde el punto de vista de la justicia.

El neoliberalismo es un tipo de capitalismo tan «indiscriminadamente promiscuo que instrumentalizaría cualquier perspectiva» (Fraser, 2015a: 260), incluida la de género. La despolitización y la individualización son completamente incompatibles con la conciencia política de un feminismo transformador. Como vemos, Fraser critica la absorción feminista del marco interpretativo típicamente neoliberal que obvia y oculta las estructuras de poder, y en su lugar, aboga por la alineación de las fuerzas

$10 \mathrm{Me}$ adscribo a una concepción abolicionista del género, según la cual el género es la marca de la opresión y el estatus. A mi juicio, en una sociedad igualitaria habría sexos, pero no género o clases sexuales. He expuesto el abolicionismo de género en García-Granero (2017) y actualmente continúo trabajando la propuesta.

11 Sobre la influencia del neoliberalismo en las identidades gay y lesbiana, cf. López Clavel (2015).

ASPARKÍA, 33; 2018, 207-223 - ISSN: 1132-8231 - DOI: HTTP:/ / DX.DOI.ORG/10.6035/ASPARKIA.2018.33.12 
de la igualdad con una protección social transformada en la batalla fundamental por afirmar el control democrático sobre unos procesos de mercantilización destructivos y desbocados. Este curso de acción conllevaría avances no sólo en la lucha por la igualdad en el eje del género, sino también en los ejes de clase y la raza.

\section{Conclusiones}

La crítica clave de Nancy Fraser al neoliberalismo radica en que no es posible integrar las reivindicaciones de redistribución, reconocimiento y representación en las sociedades neoliberales y de economía financiarizada. Las políticas neoliberales imposibilitan un cambio sustancial que transforme las sociedades en igualitarias y justas. Por ello, y en su lugar, es necesario recuperar las políticas de los Estados de Bienestar y del capitalismo gestionado por el Estado, una vez depurado de las características androcentristas con las que fueron configurados en el siglo xx.

Frente a un feminismo domesticado que deje inalteradas las distintas estructuras de poder, el feminismo resurgente ha de aprovechar la crisis de legitimación del neoliberalismo para realizar una verdadera crítica sistémica y un proyecto emancipador en el que el sueño de la liberación de las mujeres sea de nuevo parte de la visión de una sociedad solidaria. Una igualdad real entre los sexos sólo será posible en un mundo justo con todas las mujeres, gracias a un feminismo que, con una interpretación ampliada y no economicista del socialismo, incorpore conclusiones de otros movimientos, tales como el multiculturalismo, el ecologismo y el poscolonialismo.

Como hoja de ruta para que el feminismo tome un nuevo rumbo, Fraser aboga por la alianza de las feministas con otras fuerzas progresistas para crear medidas de protección y bienestar social sensibles al género. Ahora bien, dichas medidas deberán integrar tanto los parámetros de redistribución como los de reconocimiento para desmantelar eficazmente tanto las jerarquías de clase como las de estatus. Finalmente, la política feminista ha de luchar asimismo contra la ausencia de representación en los espacios transnacionales, como las agencias de las Naciones Unidas y el Foro Social Mundial. De este modo nos aproximaríamos al alcance de una paridad participativa de todas las mujeres, sin importar su situación geopolítica.

La agenda del feminismo no se ha conformar con la igualdad de oportunidades, pues ni para hombres ni para mujeres esto es suficiente. Un feminismo realmente emancipador no pretende la igualdad en una sociedad injusta. Hace falta un verdadero cambio a nivel sistémico y transitar hacia una sociedad en la que tanto hombres como mujeres tengan acceso a una vida material y culturalmente digna.

\section{REFERENCIAS BIBLIOGRÁFICAS}

Blanco Brotons, Francisco (2017). «La política del marco. Reescribiendo las fronteras de la justicia» en Revista de Filosofía, Vol. 42, No2, pp. 275-289.

Brown, Wendy (2015). Undoing the Demos: Neoliberalism's Stealth Revolution, New York: Zone. 
De Miguel, Ana (2015). Neoliberalismo sexual. El mito de la libre elección, Madrid: Cátedra. EIsEnsteIn, Hester (2005). «A Dangerous Liaison? Feminism and Corporate Globalization» en Science E Society, Vol. 69, No3, pp. 487-518.

Escalante Gonzalbo, Fernando (2016). Historia mínima del neoliberalismo, Madrid: Turner.

FrASER, Nancy (1989a). «Talking About Needs: Interpretive Contests as Political Conflicts in Welfare-State Societies» en Ethics, Vol. 99, N22, pp. 291-313.

FrAser, Nancy (1989b). Unruly Practices: Power, Discourse and Gender in Contemporary Social Theory, Minneapolis: University of Minnesota Press.

Fraser, Nancy (1993). «Clintonism, Welfare and the Antisocial Wage: The Emergence of a Neoliberal Political Imaginary» en Rethinking Marxism: A Journal of Economics, Culture \& Society, Vol. 6, No1, pp. 9-23.

FRASER, Nancy (1995). «From redistribution to recognition? Dilemmas of justice in a "postsocialist" age» en New Left Review, No212, pp. 68-93.

FRASER, Nancy (1997). Iustitia Interrupta. Reflexiones críticas desde la posición «postsocialista», Bogotá: Siglo del Hombre Editores.

FRASER, Nancy (2005). «Mapping the feminist imagination: from redistribution to recognition to representation» en Constellations, Vol. 12, No3, pp. 295-307.

FrAser, Nancy (2006). «La justicia social en la era de la política de la identidad: Redistribución, reconocimiento y participación» en FrASER, Nancy y Axel HonNETH (2006). ¿Redistribución o reconocimiento? Un debate políticofilosófico, Madrid: Ediciones Morata, pp. 16-88.

Fraser, Nancy (2008). Escalas de justicia, Barcelona: Herder.

Fraser, Nancy (2009). «Feminism, Capitalism and the Cunning of History» en New Left Review, No56, pp. 97-117.

FrASER, Nancy (2015a). Fortunas del feminismo. Del capitalismo gestionado por el Estado a la crisis neoliberal, Madrid: Traficantes de Sueños.

Fraser, Nancy (2015b). «Legitimation Crisis? On the Political Contradictions of Financialized Capitalism» en Critical Historial Studies, Vol. 2, No2, pp. 157-189.

Fraser, Nancy (2017). «The End of Progressive Neoliberalism», Dissent Maganize. Disponible en: https://www.dissentmagazine.org/online_articles/ progressive-neoliberalism-reactionary-populism-nancy-fraser (Fecha de consulta: 10/01/18).

FrAser, Nancy y BEDFORD, Kate (2008). «Social rights and gender justice in the neoliberal moment. A conversation about welfare and transnational politics» en Feminist Theory, Vol. 9, No2, pp. 225-245.

FrAser, Nancy y Gordon, Linda (1994). «A Genealogy of Dependency: Tracing a Keyword of the U.S. Welfare State» en Signs, Vol. 19, No2, pp. 309-366.

FUNK, Nanette (2013). «Contra Fraser on Feminism and Neoliberalism» en Hypatia, Vol. 28, Nº1, pp. 179-196.

GARcíA-Granero, Marina (2017). «Deshacer el sexo. Más allá del binarismo varónmujer» en Dilemata. Revista Internacional de Éticas Aplicadas, No25, pp. 253-263.

Gosse, Van (2005). Rethinking the New Left: An Interpretative History, New York: Palgrave Macmillan. 
GuerrA, María José (2011). «Nancy Fraser: la justicia como redistribución y reconocimiento» en MAíz, Ramón (Comp.) (2011). Teorías políticas contemporáneas, València: Tirant Lo Blanch, pp. 335-363.

Johnson, Pauline (2017). «Feminism as Critique in a Neoliberal Age: Debating Nancy Fraser» en Critical Horizons, Vol. 19, $\mathrm{N}^{\circ} 1$, pp. 1-17. doi: 10.1080/14409917.2017.1376937.

LEONARD, Sarah y Fraser, Nancy (2016). «Capitalism's Crisis of Care» en Dissent Magazine. Disponible en: https://www.dissentmagazine.org/article/nancyfraser-interview-capitalism-crisis-of-care (Fecha de consulta: 04/11/17).

LitTler, Jo (2013). "Meritocracy as Plutocracy: The Marketising of "Equality" under Neoliberalism» en New Formations: a journal of culture/theory/politics, No8081, pp. 52-72.

López Clavel, Pau (2015). «Tres debates sobre la hormonormativización de las identidades gay y lesbiana» en Asparkía. Investigació Feminista, No26, pp. 137-153.

Martí Gual, Ana y Poveda Rosa, María M. (2009). «El empleo de hogar: desigualdad y poder entre mujeres» en Asparkía. Investigació Feminista, No20, pp. 99-121.

MedinA-VicEnt, Maria (2018). «Flirting with Neoliberalism: The Transfiguration of Feminist Political Awareness» en NORA. Nordic Journal of Feminist and Gender Research, Vol. 25, Nº, pp. 69-75.

PALMER, Ingrid (1995). «Public Finance from a Gender Perspective» en World Development, Vol. 23, Nº11, pp. 1981-1986.

Posada Kubissa, Luisa (2015). «Justicia y género: las propuestas de Nancy Fraser» en Daimon. Revista Internacional de Filosofía, N65, pp. 7-19.

PRÜGL, Elisabeth (2014). «Neoliberalising Feminism» en New Political Economy, Vol. 20, N4, pp.614-631.

REVERTER-BAÑón, Sonia (2017). «Cosmopolitismo feminista contra globalización» en Araucaria. Revista Iberoamericana de Filosofía, Política y Humanidades, Vol. 19, No37, pp. 301-325.

RotTenberg, Catherine (2013). «The Rise of Neoliberal Feminism» en Cultural Studies, N'28, pp. 418-437.

RotTenberg, Catherine (2017). «Neoliberal Feminism and the Future of Human Capital» en Signs: Journal of Women in Culture and Society, Vol. 42, No2, pp. 329-348. VÉlez BAutista, Graciela (2009). «Conciliación entre la vida laboral y familiar de las mujeres. Un acuerdo pendiente» en Asparkía. Investigació Feminista, No20, pp. 165-183.

Recibido el 14 de enero de 2018

Aceptado el 17 de abril de 2018

BIBLID [1132-8231 (2018): 207-223] 